NASA/TM-2003-212019

AIAA-2003-0368

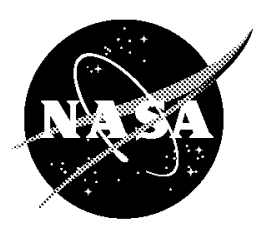

Nonlinear Resonant Oscillations of Gas in Optimized Acoustical Resonators and the Effect of Central Blockage

Xiaofan $\mathrm{Li}$, Joshua Finkbeiner, and Ganesh Raman

Illinois Institute of Technology, Chicago, Illinois

Christopher Daniels

Ohio Aerospace Institute, Brook Park, Ohio

Bruce M. Steinetz

Glenn Research Center, Cleveland, Ohio 
Since its founding, NASA has been dedicated to the advancement of aeronautics and space science. The NASA Scientific and Technical Information (STI) Program Office plays a key part in helping NASA maintain this important role.

The NASA STI Program Office is operated by Langley Research Center, the Lead Center for NASA's scientific and technical information. The NASA STI Program Office provides access to the NASA STI Database, the largest collection of aeronautical and space science STI in the world. The Program Office is also NASA's institutional mechanism for disseminating the results of its research and development activities. These results are published by NASA in the NASA STI Report Series, which includes the following report types:

- TECHNICAL PUBLICATION. Reports of completed research or a major significant phase of research that present the results of NASA programs and include extensive data or theoretical analysis. Includes compilations of significant scientific and technical data and information deemed to be of continuing reference value. NASA's counterpart of peerreviewed formal professional papers but has less stringent limitations on manuscript length and extent of graphic presentations.

- TECHNICAL MEMORANDUM. Scientific and technical findings that are preliminary or of specialized interest, e.g., quick release reports, working papers, and bibliographies that contain minimal annotation. Does not contain extensive analysis.

- CONTRACTOR REPORT. Scientific and technical findings by NASA-sponsored contractors and grantees.
- CONFERENCE PUBLICATION. Collected papers from scientific and technical conferences, symposia, seminars, or other meetings sponsored or cosponsored by NASA.

- SPECIAL PUBLICATION. Scientific, technical, or historical information from NASA programs, projects, and missions, often concerned with subjects having substantial public interest.

- TECHNICAL TRANSLATION. Englishlanguage translations of foreign scientific and technical material pertinent to NASA's mission.

Specialized services that complement the STI Program Office's diverse offerings include creating custom thesauri, building customized databases, organizing and publishing research results ... even providing videos.

For more information about the NASA STI Program Office, see the following:

- Access the NASA STI Program Home Page at http://wwzo.sti.nasa.gov

- E-mail your question via the Internet to help@stinasa.gov

- Fax your question to the NASA Access Help Desk at 301-621-0134

- Telephone the NASA Access Help Desk at 301-621-0390

- Write to: NASA Access Help Desk NASA Center for AeroSpace Information 7121 Standard Drive Hanover, MD 21076 
NASA/TM-2003-212019

AIAA-2003-0368

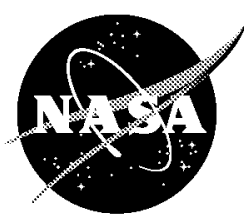

Nonlinear Resonant Oscillations of Gas in Optimized Acoustical Resonators and the Effect of Central Blockage

Xiaofan Li, Joshua Finkbeiner, and Ganesh Raman

Illinois Institute of Technology, Chicago, Illinois

Christopher Daniels

Ohio Aerospace Institute, Brook Park, Ohio

Bruce M. Steinetz

Glenn Research Center, Cleveland, Ohio

Prepared for the

41st Aerospace Sciences Meeting and Exhibit

sponsored by the American Institute of Aeronautics and Astronautics

Reno, Nevada, January 6-9, 2003

National Aeronautics and

Space Administration

Glenn Research Center 
Trade names or manufacturers' names are used in this report for identification only. This usage does not constitute an official endorsement, either expressed or implied, by the National Aeronautics and Space Administration.

The Propulsion and Power Program at

NASA Glenn Research Center sponsored this work.

Available from

NASA Center for Aerospace Information

National Technical Information Service 7121 Standard Drive 5285 Port Royal Road

Hanover, MD 21076

Springfield, VA 22100

Available electronically at http://gltrs.grcnasa.gov 


\title{
NONLINEAR RESONANT OSCILLATIONS OF GAS IN OPTIMIZED ACOUSTICAL RESONATORS AND THE EFFECT OF CENTRAL BLOCKAGE
}

\author{
Xiaofan Li, Joshua Finkbeiner, and Ganesh Raman \\ Illinois Institute of Technology \\ Chicago, Illinois 60616 \\ Christopher Daniels \\ Ohio Aerospace Institute \\ Brook Park, Ohio 44142 \\ Bruce M. Steinetz \\ National Aeronautics and Space Administration \\ Glenn Research Center \\ Cleveland, Ohio 44135
}

\begin{abstract}
Optimizing resonator shapes for maximizing the ratio of maximum to minimum gas pressure at an end of the resonator is investigated numerically. It is well known that the resonant frequencies and the nonlinear standing waveform in an acoustical resonator strongly depend on the resonator geometry. A quasi-Newton type scheme was used to find optimized axisymmetric resonator shapes achieving the maximum pressure compression ratio with an acceleration of constant amplitude. The acoustical field was solved using a one-dimensional model, and the resonance frequency shift and hysteresis effects were obtained through an automation scheme based on continuation method. Results are presented for optimizing three types of geometry: a cone, a horn-cone and a half cosine-shape. For each type, different optimized shapes were found when starting with different initial guesses. Further, the one-dimensional model was modified to study the effect of an axisymmetric central blockage on the nonlinear standing wave.
\end{abstract}

\section{INTRODUCTION}

The waveform of the standing wave in an oscillating closed cavity is strongly influenced by the geometry of the cavity (resonator). Lawrenson et al. ${ }^{1}$ at MacroSonix Corp first exploited the dependence for developing high-amplitude acoustic pressures, referred to as resonant macrosonic synthesis (RMS). Peak acoustic pressures that exceed the ambient pressure three to four times and ratios of maximum to minimum pressures of 27 were observed in RMS cavities. The size of the demonstrated overpressure reached the level that required by commercial applications such as acoustic pump or compressor. They considered these types of axisymmetric shape: cylinder, cone, hone-cone and bulb, and appeared to find that the hone-cone shape generates higher overpressure given an input power. A companion paper by Ilinskii et $a .^{2}$ developed a onedimensional time domain model for studying the RMS numerically. Their results confirmed the nonlinear standing waveform and the related characteristics such as shape-induced resonance hardening and softening observed in the experiments by Lawrenson et al. ${ }^{1}$ Chun and $\mathrm{Kim}^{2}$ numerically investigated the cosine shapes in addition to cylindrical and conical shapes using highorder finite-difference approximations. They claimed that the half cosine-shape is more suitable to induce high compression ratio than other shapes. To serve commercial needs, such as in acoustic gas compressor and acoustic liquid pump, the common goal in these studies is to find an optimized shape for generating higher overpressure. However, the optimization procedure and result have not yet been discussed.

In this article, we introduce the numerical schemes and present the results in searching for the optimal shape parameters that give us the highest maximum-tominimum pressure ratio in each of the following types of shapes: cone, horn-cone and half cosine-shape. In the second part, we study the effect of a cylindrical central blockage on the RMS

\section{GOVERNING EQUATIONS}

In this section, we describe a one-dimensional model for the acoustic wave field in the resonator that includes a central blockage. We develop our model following the work by Ilinksii et $a l^{2}$ for an axisymmetric resonator in absence of the central blockage. 
Consider the acoustic field in an oscillating resonator driven by an external force. As sketched in Fig. 1, the resonator is axisymmetric with the inner radius given by $r=r(x), 0<x<l$, where $x$ is the coordinate along the axis of symmetry. The central blockage is also axisymmetric with the same axis of symmetry $x$, whose shape is written as $r_{0}=r_{0}(x)$.

The density of the gas $\rho$, the velocity $u$ and the pressure $p$ satisfy the conservation of mass

$$
\frac{\partial \rho}{\partial t}+\frac{1}{r^{2}-r_{0}^{2}} \frac{\partial}{\partial x}\left(\left(r^{2}-r_{0}^{2}\right) \rho u\right)=0
$$

and the conservation of momentum

$$
\begin{aligned}
& \frac{\partial u}{\partial t}+u \frac{\partial u}{\partial x}=-\frac{1}{\rho} \frac{\partial p}{\partial x}-a(t) \\
& +\frac{(\xi+4 \eta / 3)}{\rho} \frac{\partial}{\partial x}\left(\frac{1}{r^{2}-r_{0}^{2}} \frac{\partial}{\partial x}\left(\left(r^{2}-r_{0}^{2}\right) u\right)\right)
\end{aligned}
$$

where $a(t)$ is the acceleration of the resonator enforced by the external force; $\xi$ and $\eta$ are coefficients of viscosity. The state equation is specified by that of an ideal gas

$$
p=p_{0}\left(\rho / \rho_{0}\right)^{\gamma}
$$

where $p_{0}$ and $\rho_{0}$ are the ambient pressure and density respectively. The no-penetration boundary conditions at the two ends require that the velocity vanish at $x=0$ and $l$.

Following Ilinksii et $a l^{2}$, expressing the variables in finite Fourier series in time, the equations (1) and (2) can be reduced to a system of Ordinary Differential Equations (ODEs) for the Fourier coefficients of the velocity potential $\varphi$, defined as $u=\nabla \varphi$ :

$$
\left\{\begin{array}{l}
\frac{d \hat{\varphi}_{k}}{d x}=\frac{\hat{v}_{k}}{r^{2}-r_{0}^{2}}, \\
\sum_{l} a_{k l} \frac{d \hat{v}_{l}}{d x}=f_{k}, k=1,2, \ldots, N
\end{array}\right.
$$

where

$$
\begin{aligned}
& v=\left(r^{2}-r_{0}^{2}\right) \frac{\partial \varphi}{\partial x}, \varphi=\sum \hat{\varphi}_{k} e^{i k t}, v=\sum \hat{v}_{k} e^{i k t}, \\
& a=\sum \hat{a}_{k} e^{i k t} \text { and } \\
& a_{k l}=a_{k l}\left(\hat{v}_{k}, \hat{\varphi}_{k}, x\right), f_{k}=f_{k}\left(\hat{v}_{k}, \hat{\varphi}_{k}, x\right) . \text { The }
\end{aligned}
$$

detailed expressions for $a_{k l}$ and $f_{k}$ are the same as those in Ilinksii et $a l^{2}$ except that the central blockage is taken into account.

$$
\begin{aligned}
& a_{k l}=\left(c_{0}^{2}+i k \delta\right) \delta_{k l}+D_{k-l}^{\prime}, \\
& f_{k}=-k^{2}\left(r^{2}-r_{0}^{2}\right) \hat{\varphi}_{k}+i k\left(r^{2}-r_{0}^{2}\right) x \hat{a}_{k}+\frac{i k\left[v^{2}\right]_{k}}{r^{2}-r_{0}^{2}}
\end{aligned}
$$$$
+\sum_{l=-N+k}^{N} \hat{a}_{k-l} \hat{v}_{k}-\frac{\frac{d\left(r^{2}-r_{0}^{2}\right)}{d x}}{\left(r^{2}-r_{0}^{2}\right)^{3}} \sum_{l=-N+k}^{N}\left[v^{2}\right]_{k-l} \hat{v}_{k},
$$

where $\delta=\frac{\zeta+4 \eta / 3}{\rho_{0}}, c_{0}$ is the small signal

propagation speed, $D_{m}^{\prime}$ is given by

$$
\begin{aligned}
D_{m}^{\prime}= & -(\gamma-1) x \hat{a}_{m}-i m(\gamma-1) \hat{\varphi}_{m} \\
& -\frac{\gamma+1}{2\left(r^{2}-r_{0}^{2}\right)^{2}}\left[v^{2}\right]_{m},
\end{aligned}
$$

and

$$
\left[v^{2}\right]_{k}=\sum_{l=-N+k}^{N} \hat{\boldsymbol{v}}_{k-l} \hat{v}_{l}
$$

The no-slip boundary conditions at the two ends are translated to the Fourier space: $\hat{v}_{k}=0$ at $x=0, l$.

After the Fourier coefficients $\left\{\hat{\varphi}_{k}\right\}$ and $\left\{\hat{v}_{k}\right\}$ are obtained by solving the boundary-value problem Eq. (4), the velocity potential $\varphi$ and the modified velocity $v$ are computed from the inverse FFT. The density $\rho$ is given by the momentum equation

$$
\frac{\rho}{\rho_{0}}=
$$

$\left(1-\frac{\gamma-1}{c_{0}^{2}}\left[\frac{\partial \varphi}{\partial t}+\frac{1}{2\left(r^{2}-r_{0}^{2}\right)^{2}} v^{2}+a x-\frac{\delta}{r^{2}-r_{0}^{2}} \frac{\partial v}{\partial x}\right]\right)^{\frac{1}{\gamma-1}}$ 
and the pressure $p$ can be obtained from the state equation $p / p_{0}=\left(\rho / \rho_{0}\right)^{\gamma}$.

\section{NUMERICAL METHODS}

In this section, we describe the procedures for finding the optimal shape parameters so that the desired standing waveform can be obtained.

To simplify our discussion, we introduce the following dimensionless variables

$$
X=\frac{x}{l}, \quad T=\omega t, \quad R=\frac{r}{l}, \quad R_{0}=\frac{r_{0}}{l}, \quad A=\frac{a}{l \omega_{0}^{2}},
$$

where $\omega$ is the frequency of the periodic force acted on the resonator, $\omega_{0}=\frac{\pi c_{0}}{l}$ is the fundamental frequency of a cylindrical resonator of length $l$.

In this work, we assume the acceleration of the resonator is harmonic, $A(T)=\tilde{A} \cos (T)$. The acoustic wave field in the resonator is determined by the acceleration amplitude $\tilde{A}$, the ratio of specific heats $\gamma$, the attenuation coefficient $G$, defined as $G=\frac{\pi(\zeta+4 \eta / 3) \omega_{0}}{c_{0}^{2} \rho_{0}}$, and the resonator oscillating frequency $\Omega$, defined as $\Omega=\frac{\omega}{\omega_{0}}$.

Now, we briefly describe the method for finding the optimal resonator shape.

In the optimization process, we fix the values of the acceleration amplitude $\tilde{A}=5 \times 10^{-4}$, the gas specific heat ratio $\gamma=1.2$ and the viscosity-related parameter $G=0.01$. Suppose the resonator shape $R(X)$ is determined by a number of shape parameters, $S_{0}, S_{1}$, $\ldots, \mathrm{S}_{\mathrm{n}}$ For example, a cone is given by $R(X)=S_{0}+S_{1} X$. The compression ratio of a resonator $\mathrm{R}_{\mathrm{c}}$, defined at the ratio of maximum pressure to the minimum pressure at the narrow end of the resonator

$$
R_{c}\left(S_{0}, S_{1, \ldots,} S_{n}\right)=\frac{p_{\max ,}}{p_{\min }} \text { at } X=0
$$

is a function of the shape parameters, the dimensionless frequency $\Omega$ and the history of $\Omega$ (due to the existence of hysteresis effects). The method for obtaining $\mathrm{R}_{\mathrm{c}}$ for fixed resonator shape is explained later in the section.

We first outline the optimization schemes. A quasiNewton method, BFGS (Broyden-Fletcher-GoldfarbShanno ${ }^{4}$ ), is used for maximizing the multi-variable nonlinear function $R_{c}\left(S_{0}, S_{1, \ldots,} S_{n}\right)$. Since the evaluation of the objective function $R_{c}$ itself involves solving a nonlinear system of ODEs Eq. (4) for many times, the gradient information of $R_{c}$ required for the BFGS method is not available analytically and is derived by partial derivatives using a numerical differentiation method via finite differences. This entails perturbing each design variables, $S_{i}$, in turn and calculating the rate of change in the objective function $R_{c}\left(S_{0}, S_{1, \ldots} S_{n}\right)$. For two shape parameters, the optimization takes 4 to 48 hours of CPU time (depending on the type of the resonator shape and initial guess for the shape parameters) on a $1.3 \mathrm{GHz}$ Athlon T-Bird PC with the Lahey-Fujitsu FORTRAN compiler.

For a given shape of the resonator and the central blockage, the boundary value problem Eq. (4) is solved numerically by a Multiple Shooting Method. Because of the hysteresis effects, the solution is not unique near the resonant frequency and the Multiple Shooting Method will not converge unless a good initial guess of the solution is provided. To circumvent the difficulty, a continuation method is implemented: the system of ODEs is solved starting from a frequency $\Omega$ that is far away from the resonant frequency and the solution is used as an initial guess for solving the ODEs for increased or decreased $\Omega$; the steps are repeated until all branches of the solution for all values of $\Omega$ near the resonance is completed. The maximum ratio $R_{c}$ among different values of $\Omega$ is chosen as the compression ratio for the resonator.

\section{RESULTS}

\section{A. Characteristics of the standing waves}

Before we present our results on finding the optimal resonator's shape, we illustrate some of the important properties of the pressure wave in a non-cylindrical resonator, whose shape is described by that of a horncone:

$$
R(X)=\left\{\begin{array}{l}
S_{0} \cosh \left(S_{1} X\right), 0 \leq X \leq S_{2} \\
\alpha+\beta X, S_{2} \leq X \leq 1
\end{array}\right.
$$


where

$S_{0}=0.028333, S_{1}=5.7264, S_{2}=0.25, \alpha=S_{0} \cosh \left(S_{1} S_{2}\right)$, and $\beta=S_{0} S_{1} \sinh \left(S_{1} S_{2}\right)$. The shape parameters for the horncone are obtained from those used in the experiments by Lawrenson et al. ${ }^{1}$

In Fig. 2, we show the pressure waveforms at the ends of cylindrical resonator and the horncone resonator at their corresponding resonance frequencies for the effective viscosity $\mathrm{G}=0.01$, the gas specific heat ratio $\gamma=1.2$, the acceleration $\widetilde{A}=10^{-3}$. Throughout this paper, we will keep the effective viscosity $G$ and the gas specific constant at these values, which are close to the values used in the experiments ${ }^{1}$ and our numerical simulations have suggested that the pressure waveform is not a strong function of these parameters. At the same level of the acceleration, the waveform at the narrow end of the horncone shows large variation in which the ratio of the maximum and minimum pressure, $R_{c}$, exceeds the value of 30.5 . On the other hand, the waveform for the cylinder shows the formation of shocks at the resonance and the compression ratio $R_{c}$ is below 1.32. The difference in the waveforms shows the strong dependence of the acoustic field on the resonator shapes. The graph also shows that the variation in pressure at the wide end of the horncone is much milder than that of the narrow end, oscillating within $23 \%$ above or below the value of the reference pressure $p_{0}$.

In Fig. 3, the ratio of the amplitudes of the second and the first harmonics of the pressure, $p_{2} / p_{1}$, are plotted against the reduced amplitude of the first harmonic, $p_{1} / p_{0}$, calculated at one end of the cylinder and at the narrow end of the horncone with increasing frequency. The figure shows that the second harmonic reaches its maximum at small amplitude of the first harmonic for the consonant (cylinder) resonator and the second harmonic increases slowly as the first harmonic increases for the dissonant (horncone) resonator. In other words, the energy is pumped into the first harmonic more for the horncone than that for the cylinder, creating the large-amplitude pressure wave in the horncone resonator. In contrast, the energy is efficiently absorbed by all harmonics of the pressure wave in a cylindrical resonator and the amplitudes of the harmonics reach their maximums at small amplitude of the pressure.

In Fig. 4, the amplitude of the first harmonic is plotted against the frequency of the oscillating resonator for two different levels of acceleration: $\widetilde{A}=2 \times 10^{-4}$ and $\widetilde{A}=10^{-3}$. For the smaller acceleration, the pressure is uniquely determined at each frequency; for the larger drive amplitude, the pressure takes one of the multiple values near the resonance, depending on the direction of the change in frequency. The existence of hysteresis and hardening resonance in horncone resonators requires that the largest pressure compression ratio be obtained through an upward frequency sweep. Our numerical simulations show that the frequency increment size near the resonance must be small for the convergence of the Multiple Shooting method.

\section{B. Optimal conical shapes}

In following subsections, we present results on optimizing resonator shapes to achieve maximum compression ratio $R_{c}$ at one end of the resonators. For simplicity and reducing computing cost, we assume the central blockage is absent, i.e., $R_{0}=0$, and keep ratio of specific heats $\gamma=1.2$, the attenuation coefficient $G=0.01$ and the acceleration $\tilde{A}=5 \times 10^{-4}$ constant.

A conical resonator can be written as

$$
R(X)=S_{0}+S_{1} X, \quad \text { for } \quad 0 \leq X \leq 1
$$

Using the optimization procedure described in Sec. II and starting with the conical shape given in Lawrenson et al., ${ }^{1}$ we found that the compression ratio $R_{c}$ of a conical resonator reaches a maximum when the cone has the shape constants: $S_{0}=0.032945$ and $S_{1}=0.26800$. The achieved compression ratio is $R_{c}=5.048$ for the fixed values of the parameters given earlier. Recall that, due to hysteresis, in order to find $R_{c}$, one has to trace out the entire branch by incrementing the frequency $\Omega$. The compression ratio reaches the value when the frequency is increased to 1.3135 . The conical resonator studied in Lawrenson et al. ${ }^{1}$ and Ilinksii et al. ${ }^{2}$ has almost identical shape $S_{0}=0.032941$ and $S_{1}=0.26800$.

The adopted optimization scheme BFGS is designed for finding a local extreme of a multivariable function. Our numerical simulations indicate that the compression ratio, as a function of the shape parameters, have many local extrema. Starting with a different initial shape, we obtained another locally optimized conical shape with $S_{0}=0.010413$ and $S_{1}=0.19572$. When the frequency is raised to the value of 1.3862 by small increments, the compression ratio reaches the value of 5.343 , which is about $6 \%$ higher than that for the first optimal conical resonator. In the current optimization, the slope of the cone is restricted to be less than 0.268 and we require that the radius at the small end be larger than 0.01 . The 
two optimal conical resonators are shown in Fig. 5. The second cone (Fig. 5(b)) has the smaller narrow end and smaller slope than the first one (Fig. 5(a)).

\section{Optimal horn-cone shapes}

Horn-cone geometry is described by

$$
R(X)=\left\{\begin{array}{lcc}
R_{h} \cosh \left(D_{h} X\right) & \text { for } & 0 \leq X \leq X_{c} \\
\alpha+\beta\left(X-X_{c}\right) & \text { for } & X_{c} \leq X \leq 1
\end{array}\right.
$$

where

$$
\alpha=R_{h} \cosh \left(D_{h} X_{c}\right) \text { and } \beta=R_{h} D_{h} \sinh \left(D_{h} X_{c}\right)
$$

$R_{h}, X_{c}$ and $D_{h}$ are positive constants. We consider that the connection point of horn and cone in the horncone is fixed at $X_{c}=0.25$. We optimize the remaining two shape parameters $S_{0} \equiv R_{h}$ and $S_{1} \equiv D_{h}$. We found that the dimensions of the horn-cone given in Lawrenson et al. ${ }^{1}, R_{h}=0.028333, D_{h}=5.7264$, are nearly locally optimized if we start the optimization with these values. For these parameters, the maximum compression ratio 13.10 is achieved when the frequency is increased to 1.4671 . In searching the optimal horncone design, the parameters, such as acceleration and specific heat ratio, are fixed at the values given in the beginning of Sec. III B. Starting with a different initial geometry of the horncone, we can obtain a higher value of the compression ratio $R_{c}=17.12$ when $R_{h}$ $=0.024488, D_{h}=5.1434$. For the compression ratio, the frequency was increased to 1.4110 gradually with small increments. Comparing with the compression ratio for the horncone in Lawrenson et al. ${ }^{1}$ (shown in Fig. 6(a)), the second optimal horncone (shown in Fig. 6(b)) improved the ratio by more than $30 \%$ at the same value of acceleration. Shown in Fig. 6(c), the pressure waveform at the narrow end for new horncone design is a little more complicated than that for the original horncone given Lawrenson et al. ${ }^{1}$

\section{Optimal $1 / 2-$ cosine shape}

We define a $1 / 2$-cosine shape as

$$
R(X)=S_{0}+S_{1}(1-\cos (\pi X), \text { for } 0 \leq X \leq 1 .
$$

The parameters of the resulting optimal cosine shape are $S_{0}=0.016307, S_{1}=0.093041$, shown in Fig. 7(b), compared with $S_{0}=0.025, S_{1}=0.095$, the $1 / 2$-cosine dimensions reported in Chun and $\mathrm{Kim}^{3}{ }^{3}$ For the optimal shape, we obtain the compression ratio $R c=10.67$ at the frequency $\Omega=1.5683$, which is about $11 \%$ better than that of Chun and $\mathrm{Kim}^{3}$ at the same level of acceleration. Under the specified conditions, we found that horncone shape is better than the cosine shape in generating higher compression ratio at the narrow end, which is opposite to the findings of Chun and Kim. ${ }^{3}$ Shown in Fig. 7(c), the pressure waveform of the optimal $1 / 2$-cosine design is smoother than that of Chun and $\mathrm{Kim}^{3}$

\section{E. The effect of the central blockage}

In this section, we discuss the influence of the central blockage on the pressure waveforms in a resonator of fixed shape. In this work, we limit ourselves to cylindrical central blockage, though our equations derived in Sec. I apply to arbitrary axisymmetric shape of central blockage.

Figure 8 shows the pressure waves at the narrow end of a conical resonator described by Eq. (8) with $S_{0}=0.032945$ and $S_{1}=0.26800$ for different central blockage radius sizes: $R_{0}=0,0.01$ and 0.02 . These pressure profiles were obtained at the fixed frequency $\Omega=1.3132$ and for $\tilde{A}=5 \times 10^{-4}, G=0.01, \gamma=1.2$.

The central blockage in this case was a simple cylinder. It appears that the presence of a central blockage reduces the amplitude of the pressure wave. However, Fig. 9 shows the resonant frequency of the conical resonator with a central blockage is shifted. Keeping other parameters constant, the pressure waveforms for the conical resonator with the cylindrical blockage of radius $R_{0}=0.02$, at different oscillating frequencies $\Omega=$ 1.2812, 1.3068 and 1.3291, are shown in Fig. 9.

Comparing to the pressure wave shown in Fig. 8 for a resonator without a central blockage, the amplitude of the pressure is nearly the same when the central blockage of radius 0.02 is included. The results indicate that a cylindrical central blockage in a conical resonator would affect the resonant frequency but would have negligible effect on the amplitude and overall shape of the pressure wave at the resonant frequency of the resonator/blockage system.

\section{CONCLUSION}

We have performed local optimization schemes for finding the resonator shapes that maximize the pressure compression ratio at one end of the resonators. The dimensions for several types of optimal resonators are reported: cone, horncone and $1 / 2$-cosine shapes. For each type, we found there are many different designs that achieve local extrema. Trying with different initial guesses for the optimal design, we show that one can 
get as much as 30\% improvement on the compression ratio with a fixed level of acceleration. For the shapes we considered, it appears that the horncone shape generates the highest compression ratio. Strategies for searching globally optimal shapes are under investigation.

The effect of including a cylindrical central blockage in a resonator is studied in this work as well. For a conical resonator, it seems that the resonance frequency is increased when a central blockage is present and the compression ratio at resonance is not significantly affected.

\section{REFERENCES}

${ }^{1}$ Lawrenson, C.C., Lipkens, B., Lucas, T.S., Perkins, D.K., and Van Doren, T.W., "Measurements of macrosonic standing waves in oscillating closed cavities," J. Acoust. Soc. Am. Vol. 104(2), Pt. 1, pp. 623-636, August 1998.

${ }^{2}$ Ilinskii, Y.A., Lipkens, B., Lucas, T.S., Van Doren, T.W., and Zabolotskaya, E.A., "Nonlinear standing waves in an acoustical resonator," J. Acoust. Soc. Am. Vol. 104(5), pp. 2664-2674, November 1998.

${ }^{3}$ Chun, Y.-D. and Kim Y.-H., "Numerical analysis for nonlinear resonant oscillations of gas in axisymmetric closed tubes," J. Acoust. Soc. Am. Vol. 108(6), pp. 2765-2774, December 2000.

${ }^{4}$ Gill, P.E., Murray, W., and Wright, M.H., Practical

Optimization (Academic Press, New York, 1981).

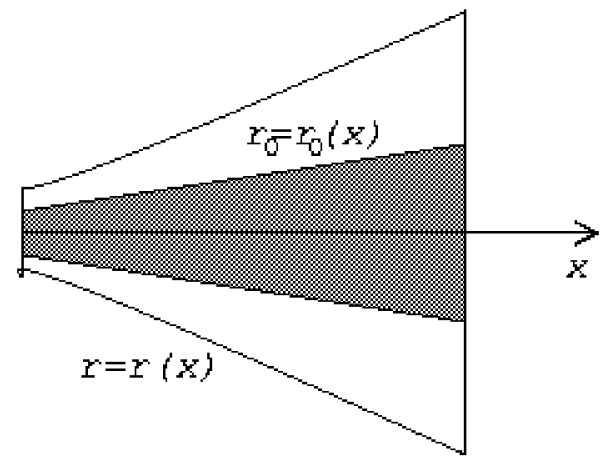

Figure 1. Sketch of an axisymmetric resonator and a central blockage.

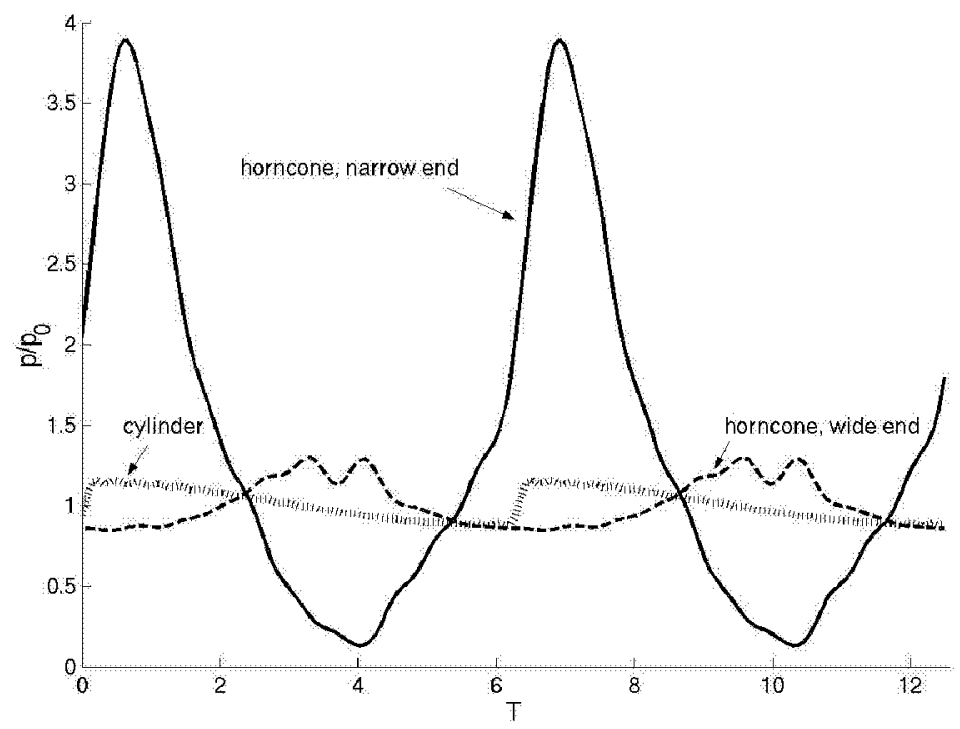

Figure 2. The reduced pressure $p / p_{0}$ at the ends of a horncone and a cylinder at their corresponding resonance frequencies for the same acceleration $\widetilde{A}=10^{-3}$. 


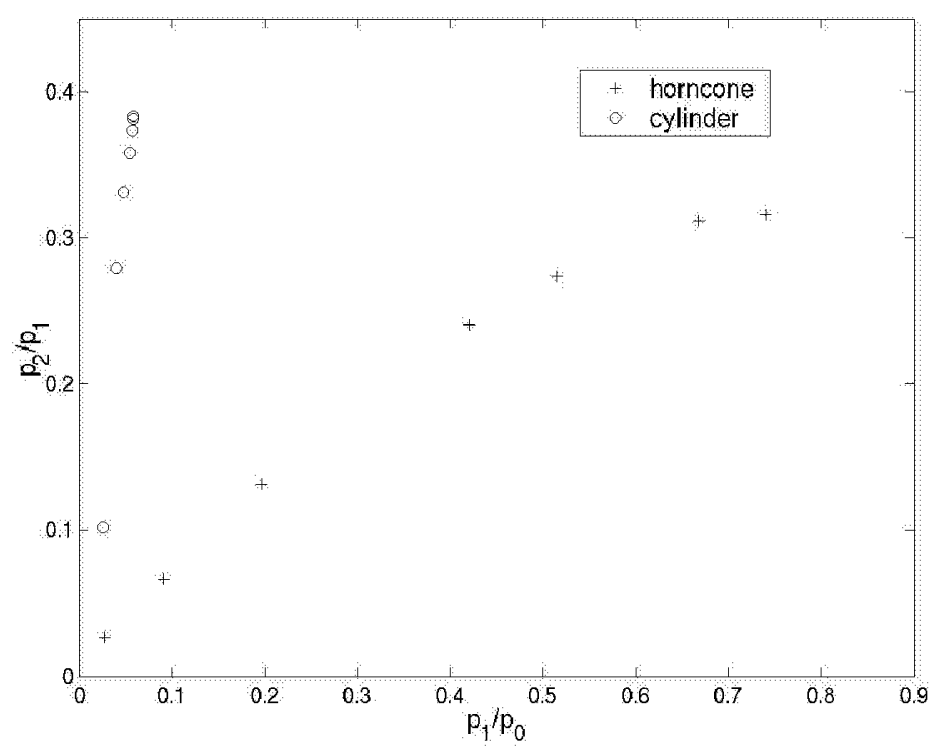

Figure 3. The ratio between the amplitudes of the second and the first harmonics of the pressure wave, $p_{2} / p_{1}$, is plotted as a function of the dimensionless amplitude of the first harmonic reduced pressure $p_{1} / p_{0}$. With increasing frequency, these amplitudes are calculated for the narrow end of the horncone and at one end of the cylinder.

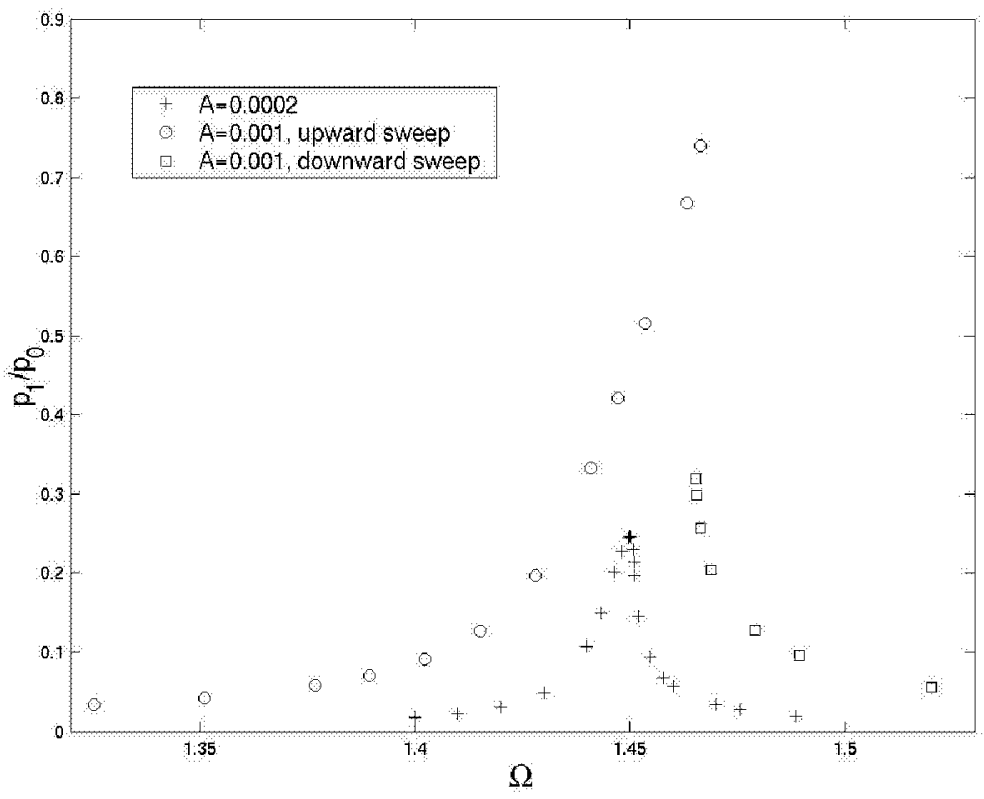

Figure 4. The effect of the frequency and the amplitude of acceleration on the dimensionless amplitude of the first harmonic reduced pressure $p_{1} / p_{0}$. The pressure is calculated for the narrow end of the horncone for the acceleration $\widetilde{A}=2 \times 10^{-4}$ (crosses) and $\widetilde{A}=10^{-3}$ (circles and squares). 
(a)

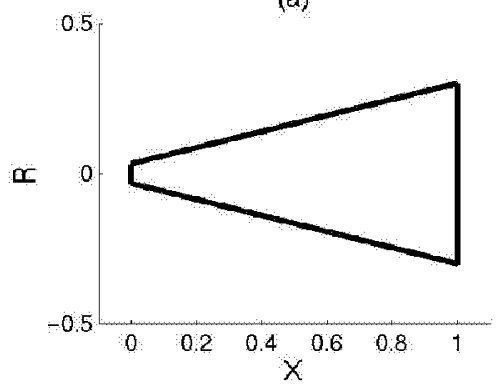

(b)

$\ddot{\alpha}$

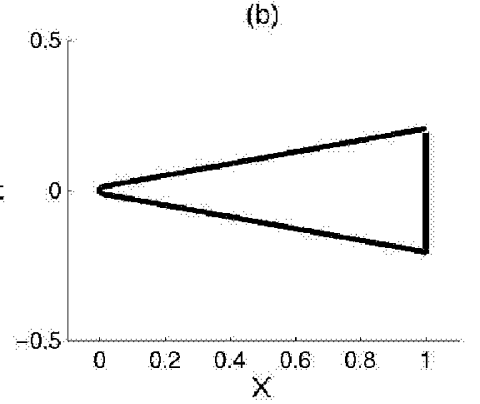

Figure 5. Optimized conical shapes: the compression ratios corresponding to the shapes in (a) and (b) reach the values of 5.048 and 5.343 respectively. For shape parameters, see the text.
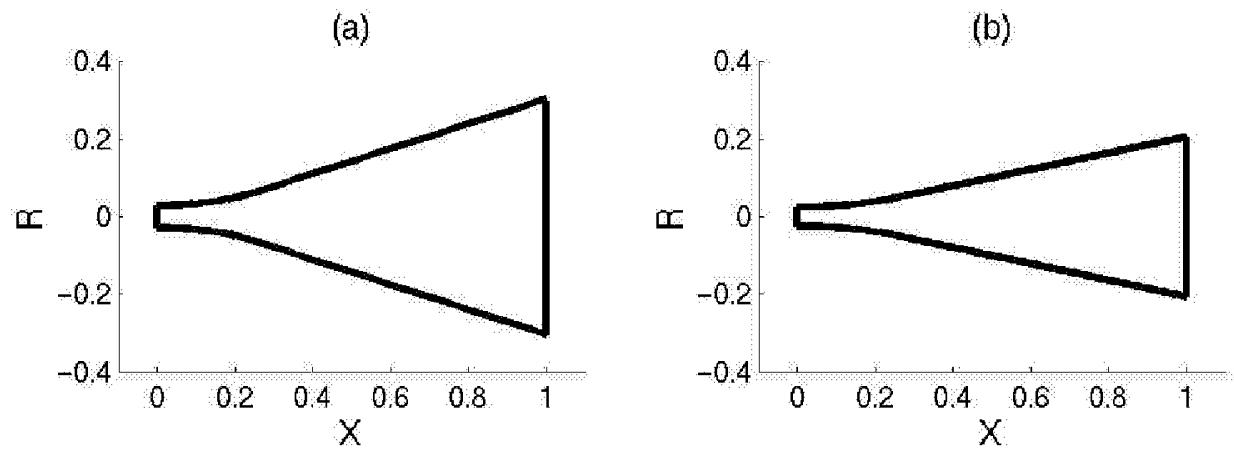

(c)

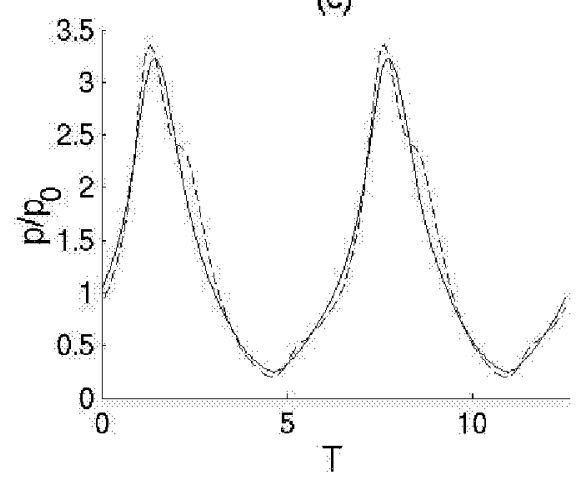

Figure 6. (a) The horncone shape in Lawrenson et al. ${ }^{1}$ (b) Optimized horncone shape. For shape parameters, see the text. (c) The pressure waveform at the narrow end for the horncone in Lawrenson et al. ${ }^{1}$ (the solid line) and that for the second optimized horncone (the dashed line) are shown. 
(a)

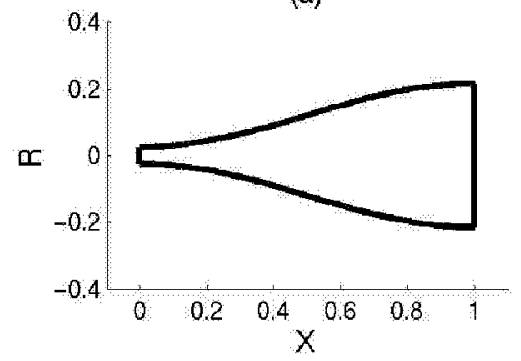

(c)

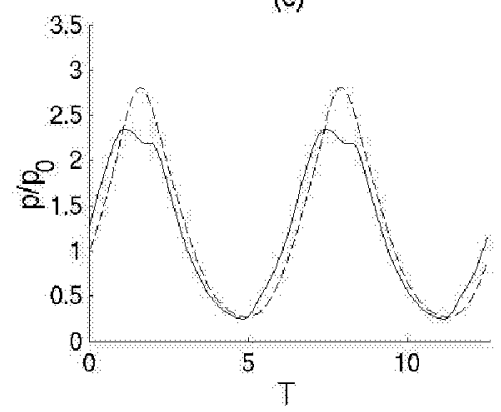

(b)

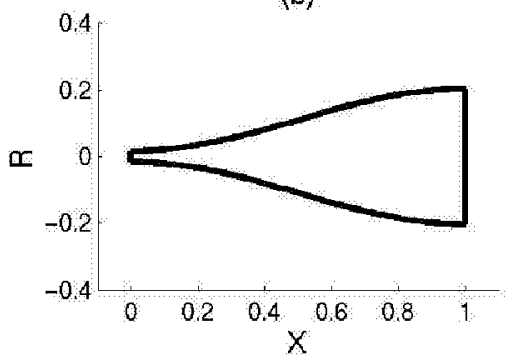

Figure 7. (a) The $1 / 2$-cosine shape as in Chun and $\mathrm{Kim}^{3}$. (b) The optimized 1/2-cosine shape. (c) The pressure waveform at the narrow end for the $1 / 2$-cosine resonator in Chun and $\mathrm{Kim}^{3}$ (the solid line) and that for the optimized $1 / 2$-cosine shape (the dashed line) are shown.

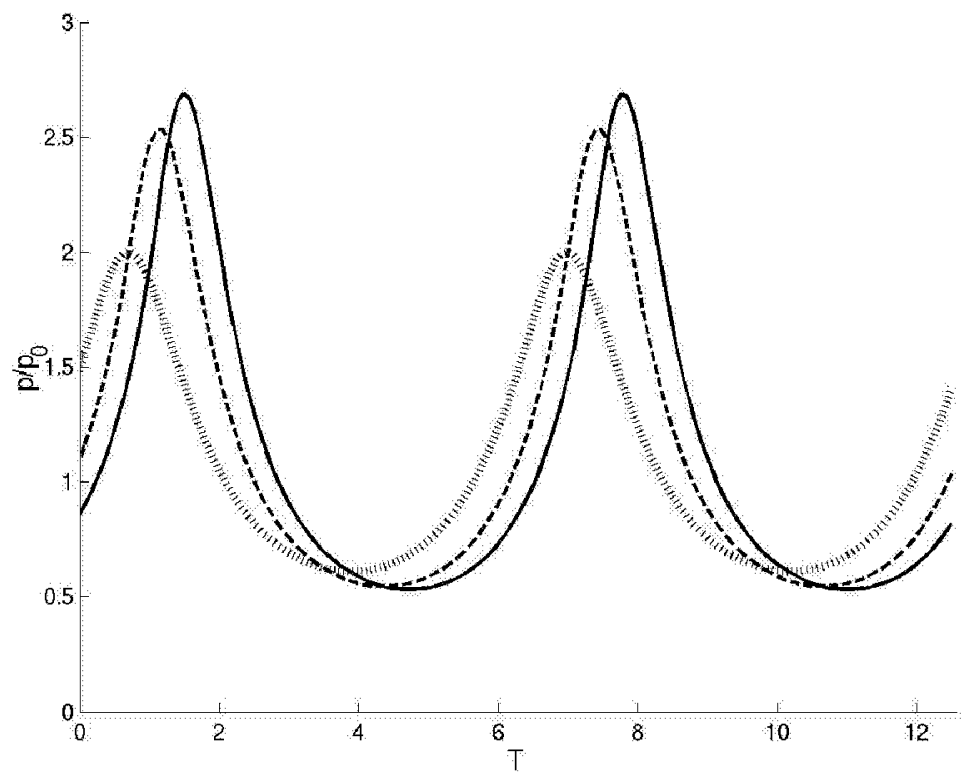

Figure 8. Effect of the center blockage. The pressure waveforms at the narrow end of a cone resonator are plotted for the different radii of the central blockage: $R_{0}=0$ (solid line), $R_{0}=0.01$ (dashed line) and $R_{0}=0.02$ (dotted line). The parameters corresponding to the plot are given by $\Omega=1.3132, \tilde{A}=5 \times 10^{-4}, G=0.01, \gamma=1.2$. 


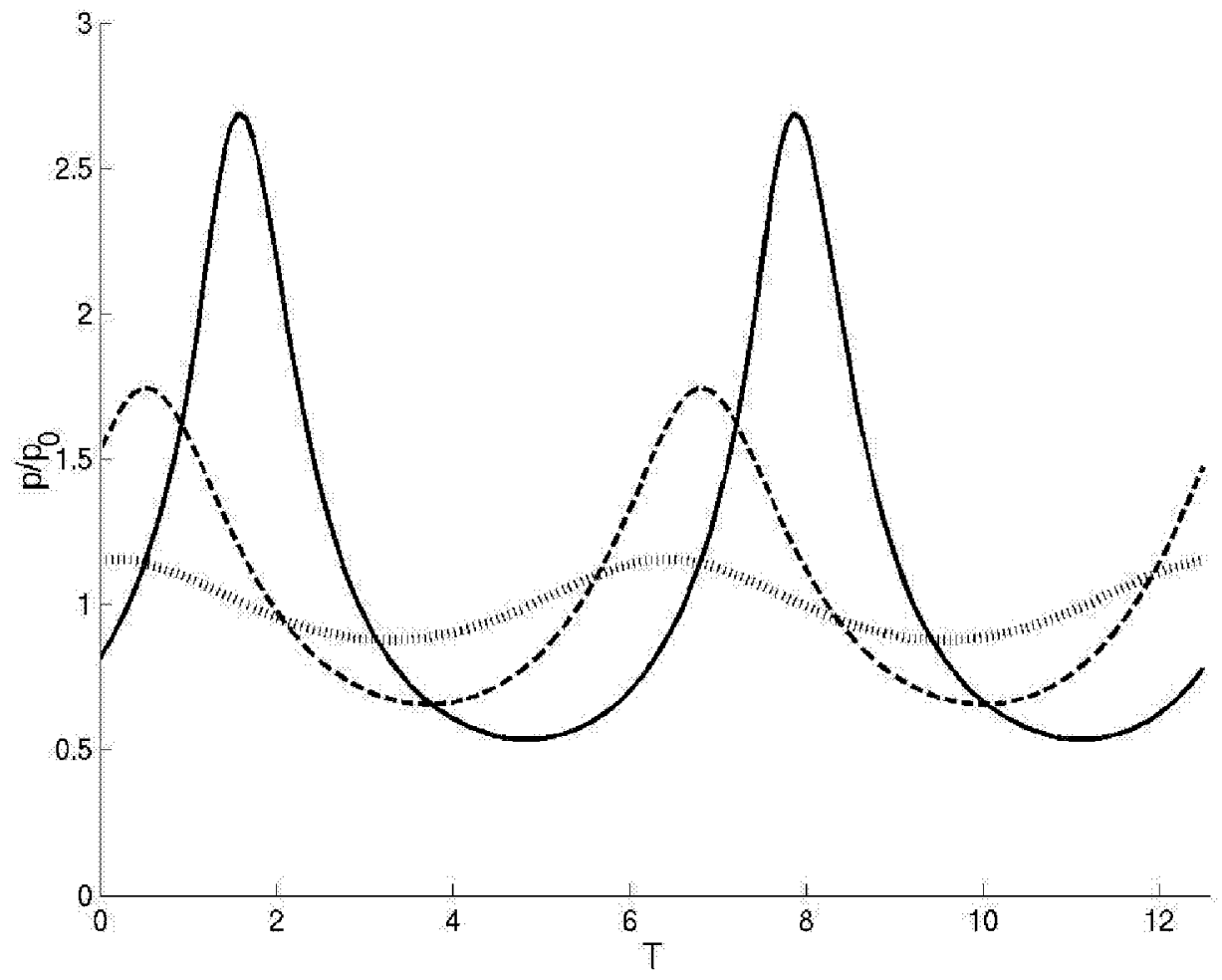

Figure 9. Pressure waveform at different frequencies: $\Omega=1.2812$ (the dotted line), $\Omega=1.3068$ (the dashed line) and $\Omega=1.3291$ (the solid line), for a conical resonator with the cylindrical central blockage of radius $R_{0}=0.02$.

The rest of the parameters corresponding to the plot are given by $\tilde{A}=5 \times 10^{-4}, G=0.01, \gamma=1.2$. 


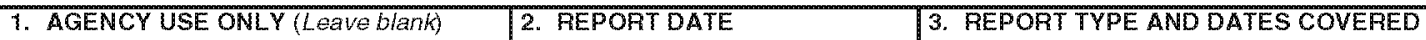

Nonlinear Resonant Oscillations of Gas in Optimized Acoustical Resonators and the Effect of Central Blockage

6. A.BTHOR(S)

5. FUNDING NUMBERS

Xiaofan Li, Joshua Finkbeiner, Ganesh Raman, Christopher Daniels, and Bruce M. Steinetz

\section{PERFORMING ORGANIZATION NAME(S) AND ADDRESS(ES)}

National Aeronautics and Space Administration

John H. Glenn Research Center at Lewis Field

Cleveland, Ohio 44135-3191

WBS-22-708-28-15

\section{SPONSORING/MONITORING AGENCY NAME(S) AND ADDRESS(ES)}

National Aeronautics and Space Administration

Washington, DC 20546-0001

10. SPONSORING/MONITORING AGENCY REPORT NUMBER

NASA TM--2003-212019

AIAA- -2003 -0368

\section{SUPPLEMENTARY NOTES}

Prepared for the 41st Acrospace Sciences Meeting and Exhibit sponsored by the American Institute of Aeronautics and Astronautics, Reno, Nevada, January 6-9, 2003. Xiaofan Li, Joshua Finkbeiner, and Ganesh Raman, Mlinois Institute of Technology, Chicago, llinois 60616; Christopher Daniels, Ohio Aerospace Institute, Brook Park, Ohio 44142; and Bruce M. Steinetz, NASA Glenn Research Center. Responsible person, Bruce M. Steinetz, organization code 5950 , $216-433-3302$.

12a. DISTRIBUTION/AVAILABILITY STATEMENT

Unclassified-Unlimited

Subject Category: 71

Distribution: Nonstandard

Available electronically at mo/gitrs.scinasiagow

This publication is available from the NASA Center for AeroSpace Information, 301-621-0390.

\section{ABSTRACT (Maximum 200 words)}

Optimizing resonator shapes for maximizing the ratio of maximum to minimum gas pressure at an end of the resonator is investigated numerically. It is well known that the resonant frequencies and the nonlinear standing waveform in an acoustical resonator strongly depend on the resonator geometry. A quasi-Newton type scheme was used to find optimized axisymmetric resonator shapes achieving the maximum pressure compression ratio with an acceleration of constant amplitude. The acoustical field was solved using a one-dimensional model, and the resonance frequency shift and hysteresis effects were obtained through an automation scheme based on continuation method. Results are presented for optimizing three types of geometry: a cone, a horn-cone, and a half cosine-shape. For each type, different optimized shapes were found when starting with different initial guesses. Further, the one-dimensional model was modified to study the effect of an axisymmetric central blockage on the nonlinear standing wave.

\section{SUBJECT TERMS}

Acoustic resonance; Cavity resonators; Oscillators; Sound generators; Standing waves

17. SECURITY CLASSIFICATION OF REPORT

Unclassified

18. SECURITY CLASSIFICATION
OF THIS PAGE
Unclassified

Unclassified

\section{SECURITY CLASSIFICATION} OF ABSTRACT

Unclassified
15. NUMBER OF PAGES

16

16. PRICE CODE

\section{LMITATION OF ABSTRACT}

